\title{
HOW MANY INFRARED DARK CLOUDS CAN FORM MASSIVE STARS AND CLUSTERS?
}

\author{
Jens Kauffmann ${ }^{1,3}$ and Thushara Pillai ${ }^{2,4}$ \\ ${ }^{1}$ Jet Propulsion Laboratory, California Institute of Technology, 4800 Oak Grove Drive, Pasadena, CA 91109, USA; jens.kauffmann@jpl.nasa.gov \\ ${ }^{2}$ Caltech Astronomy Department, 1200 East California Blvd., Pasadena, CA 91125, USA \\ Received 2010 April 15; accepted 2010 September 16; published 2010 October 7
}

\begin{abstract}
We present a new assessment of the ability of Infrared Dark Clouds (IRDCs) to form massive stars and clusters. This is done by comparison with an empirical mass-size threshold for massive star formation (MSF). We establish $m(r)>870 M_{\odot}(r / p c)^{1.33}$ as a novel approximate MSF limit, based on clouds with and without MSF. Many IRDCs, if not most, fall short of this threshold. Without significant evolution, such clouds are unlikely MSF candidates. This provides a first quantitative assessment of the small number of IRDCs evolving toward MSF. IRDCs below this limit might still form stars and clusters of up to intermediate mass, though (like, e.g., the Ophiuchus and Perseus Molecular Clouds). Nevertheless, a major fraction of the mass contained in IRDCs might reside in few $10^{2}$ clouds sustaining MSF.
\end{abstract}

Key words: ISM: clouds - methods: data analysis - stars: formation

Online-only material: color figures

\section{INTRODUCTION}

About a decade ago, Galactic plane surveys revealed large numbers of Infrared Dark Clouds (IRDCs; Egan et al. 1998; Perault et al. 1996). These are identified as dark patches against the diffuse Galactic mid-infrared background. First studies of very opaque IRDCs suggested that these have very high densities, column densities, and masses $\left(n\left[\mathrm{H}_{2}\right] \gtrsim 10^{5} \mathrm{~cm}^{-3}\right.$, $N\left[\mathrm{H}_{2}\right] \gtrsim 10^{23} \mathrm{~cm}^{-2}, m \gtrsim 10^{3} M_{\odot}$; Carey et al. 1998). Since they are dark, they are likely to be in an early evolutionary phase. Embedded in IRDCs are "cores" of a few dozen solar masses (Carey et al. 2000). It has therefore been suggested that many IRDCs are the long-sought examples of clouds just at the onset of the formation of massive stars and (proto-)clusters. This notion was corroborated by observations of young massive stars in a few individual IRDCs (Rathborne et al. 2005, 2007; Pillai et al. 2006; Beuther \& Steinacker 2007). Such views also form the framework of schemes for IRDC evolution (e.g., Rathborne et al. 2006; Rygl et al. 2010) and reviews (e.g., Menten et al. 2005; Beuther et al. 2007). IRDC samples are usually compared to regions of massive star formation (MSF) such as Orion and M17 (e.g., Ragan et al. 2009).

This picture cannot be complete, though. The above studies (and Peretto \& Fuller 2009) acknowledge that regions forming low- and intermediate-mass stars can also appear as shadows in images at mid-infrared wavelength (Abergel et al. 1996). Such IRDCs will not form massive stars. Unfortunately, the number of IRDCs evolving toward MSF is presently not known. Fractions up to $100 \%$ have been considered in the past (Section 4.3).

In this Letter, we thus use a novel criterion to provide the first conclusive quantitative demonstration that only few IRDCs are headed toward MSF. This aids the identification of pre-MSF IRDCs as targets for ALMA and Herschel. As a bonus, the MSF threshold identified below-the first observational limit of this kind-informs theory.

\footnotetext{
3 NPP Fellow.

4 CARMA Fellow.
}

In Papers I and II (Kauffmann et al. 2010a, 2010b), we show that solar neighborhood clouds devoid of MSF (specifically: Perseus, Ophiuchus, Taurus, and Pipe Nebula) generally obey

$$
m(r) \leqslant 870 M_{\odot}(r / \mathrm{pc})^{1.33} .
$$

IRDCs submitting to Equation (1) would resemble, e.g., Ophiuchus and Perseus, but not Orion (which violates Equation (1)). Figure 1 illustrates why clouds bound for MSF must exceed Equation (1). Since star formation necessitates an appropriate mass reservoir, MSF requires that a large mass is concentrated in a relatively small volume. Based on more detailed theoretical considerations, Section 4.1 puts quantitative limits on this intuitively evident reasoning. As seen in Figure 1, the masses in this MSF region are well above the mass-size range bound by Equation (1). Observations of MSF clouds confirm Equation (1) as a true MSF limit (Section 3.1). This suggests the use of Equation (1) to roughly separate IRDCs with (future) MSF from those without.

This Letter is organized as follows. Based on data from Section 2, Section 3.1 confirms (using known MSF clouds) that Equation (1) approximates an MSF limit. Many well-studied IRDCs $(25 \%-50 \%)$ fall short of this threshold (Section 3.2). Less certain data for complete IRDC samples suggest that most IRDCs obey Equation (1) and will thus not form massive stars (Section 3.3). Still, most of the mass contained by IRDCs might be in clouds forming massive stars (i.e., those violating Equation (1)).

\section{METHOD AND DATA}

\subsection{Sample}

Data for solar neighborhood clouds not forming massive stars (here: Taurus, Perseus, Ophiuchus, and Pipe Nebula) are taken from Paper II (and references therein). We rely on bolometer surveys to characterize MSF sites: Beuther et al. (2002) study FIR color-selected MSF candidates with CS-detected dense gas but no radio continuum; Mueller et al. (2002) map water masers embedded in CS clumps of high bolometric luminosity 


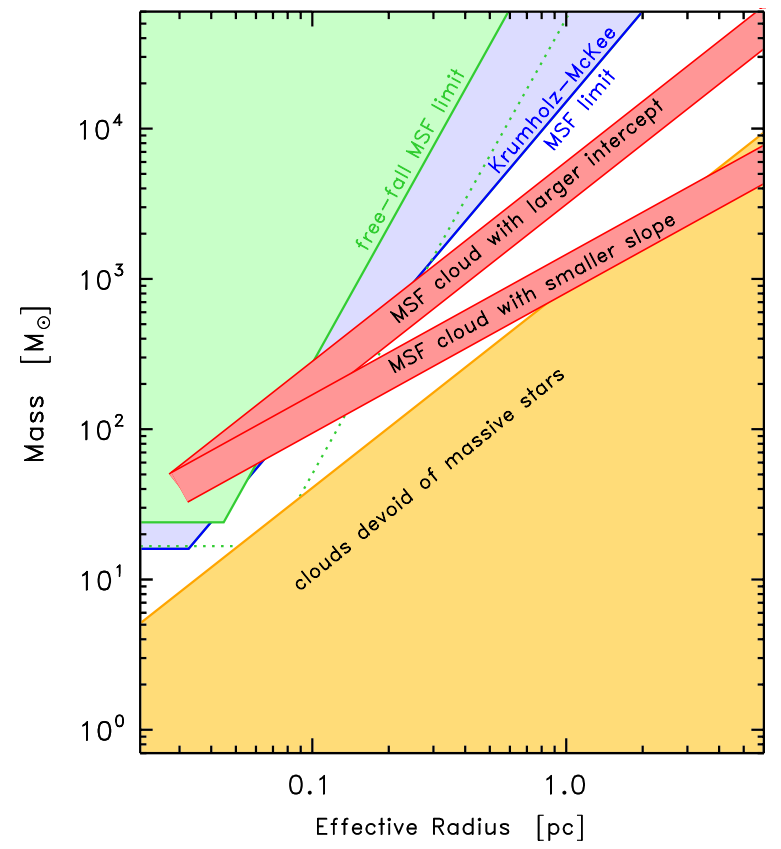

Figure 1. Plausible theoretical mass-size limits for massive star formation (MSF, Section 4.1; green and blue shading) in relation to mass-size laws (e.g., $m[r]=m_{0} \cdot r^{b}$ ) observed for non-MSF clouds (Equation (1), Figure 2; yellow shading). At small radii, MSF clouds (highlighted in red) must contain fragments bound by the theoretical MSF limits. Depending on the interplay of slope, $b$, and intercept, $m_{0}$, such clouds must also at radii $\gtrsim 0.1 \mathrm{pc}$ be more massive than fragments in non-MSF clouds.

(A color version of this figure is available in the online journal.)

$\left(>10^{3} L_{\odot}\right)$; Hill et al. (2005) explore methanol masers and ultracompact Hir regions; Motte et al. (2007) study the nearby Cygnus-X MSF site (we use their "clumps"). To exclude fragments that are not forming massive stars, we only use the "Type 1" sources $\left(\mathrm{CH}_{3} \mathrm{OH}\right.$ and/or $\mathrm{CH}_{3} \mathrm{CN}$ emission, no resolved radio continuum) from the Beuther et al. (2002) survey and ignore the secondary "mm-only" cores (without masers and Hir regions) in the Hill et al. (2005) study.

The IRDC samples were created using Midcourse Space Experiment and Spitzer images. Rathborne et al. (2006; using bolometers) and Ragan et al. (2009; using $8 \mu \mathrm{m}$ extinction) focus on clouds with stark $8 \mu \mathrm{m}$ contrast. Simon et al. (2006) report ${ }^{13} \mathrm{CO}$-based results for all IRDCs evident in their ${ }^{13} \mathrm{CO}$ Galactic Plane Survey. Peretto \& Fuller (2009) catalog extinction properties for 11,000 Spitzer $8 \mu \mathrm{m}$ IRDCs with unknown distances.

\subsection{Data Processing}

The mass-size data for solar neighborhood clouds are derived in Paper II (using methods summarized in Section 2.1 and Figure 1 of Paper I). They are based on column density maps derived from dust emission (MAMBO and Bolocam) and extinction (Two Micron All Sky Survey (2MASS)) data. Using a dendrogram method introduced by Rosolowsky et al. (2008), starting from a set of local column density maxima, a given column density map is contoured with infinitesimal level spacing. Every contour defines the boundary of a cloud fragment. We derive the contour-enclosed mass and the effective radius, $r=(A / \pi)^{1 / 2}$. Subsequent contours/fragments are usually nested. This defines relationships between cloud fragments, essentially yielding series of mass-size measurements. In Figure 2, such series are drawn using continuous lines.
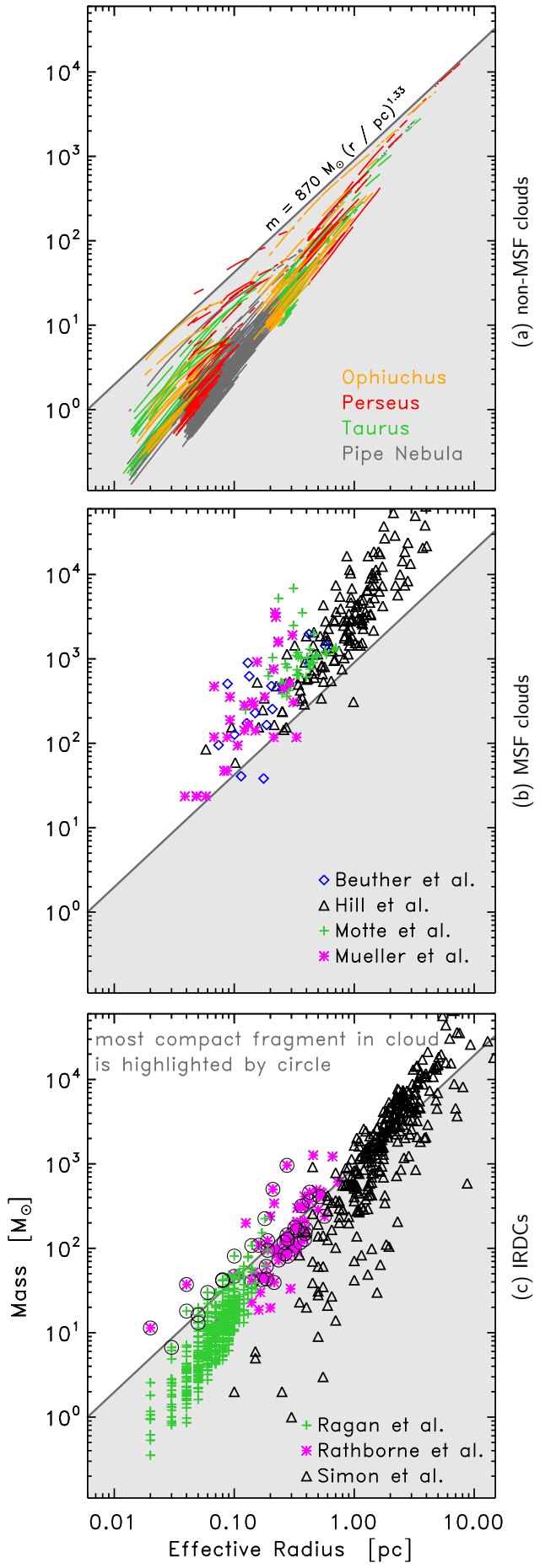

Figure 2. Clouds with (panel (b)) and without MSF (panel (a)), compared to IRDCs (panel (c)). Conceptually, data in panels (b) and (c) correspond to picking a single mass-size measurement along one of the lines shown in panel (a). The MSF and non-MSF clouds suggest that the indicated limiting power law (Equation (1)) approximates a mass-size limit for MSF (Section 3.1). Only a fraction of the IRDCs exceed this MSF limit (Figure 3, Section 3.2). If a star-forming region contains more than one fragment (i.e., clump, core, etc.), the most compact fragment (i.e., with maximum $m[r] / m_{\lim }[r]$ ) is highlighted by a circle.

(A color version of this figure is available in the online journal.)

To derive column densities from the extinction maps, we assume that column density and visual extinction are related by $N_{\mathrm{H}_{2}}=9.4 \times 10^{20} \mathrm{~cm}^{-2}\left(A_{V} / \mathrm{mag}\right)$ (Bohlin et al. 1978). To combine dust emission and extinction observations, they 
must be calibrated with respect to one another. In practice, we use Ossenkopf \& Henning (1994) dust opacities (decreased by a factor 1.5 , to match observed opacity laws; Section 4.2 of Paper I) for emission-based masses.

For comparisons, we must scale all masses to the column density laws from Paper II. Also, it is necessary to harmonize the different definitions of mass and size. The scaled data are shown in Figure 2.

Where relevant, we use dust temperatures suggested by the original studies. However, we substitute our choice of dust opacities and the aforementioned 1.5 scaling factor. ${ }^{13} \mathrm{CO}$ masses are directly taken from Simon et al. (2006), since their ${ }^{13} \mathrm{CO}$-to-mass conversion law is in rough agreement with (i.e., by factors of 1.1-2.0 larger than) the extinction-calibrated ones derived by Pineda et al. (2008). We assume that dust emission at $1.2 \mathrm{~mm}$ wavelength and optical depth at $8 \mu \mathrm{m}$ wavelength are related by $F_{v}^{\text {beam }}=50 \mathrm{mJy}\left(11^{\prime \prime} \text { beam }\right)^{-1} \cdot \tau_{8 \mu \mathrm{m}}$ (Equation (4) of Peretto \& Fuller 2009) and derive column densities from these intensities (assuming dust at $15 \mathrm{~K}$, and using the 1.5 scaling factor). We thus increase the Ragan et al. (2009) masses (from their case "A") by a factor of 1.47 (to account for their choice of opacities and molecular weights). ${ }^{5}$

In many cases (Beuther et al. 2002; Hill et al. 2005; Rathborne et al. 2006; Motte et al. 2007), the size listed in the original publication refers to the contour at half-peak intensity, while the mass measurement includes emission at much lower levels. In these cases, we assume that the sources have a near-Gaussian shape (just as explicitly assumed in many of the original papers). For such sources, the mass contained in the half-peak column density contour is just a fraction $\ln (2) \approx 0.69$ of the total mass (Equation (A.23) of Kauffmann et al. 2008; the area at halfpeak intensity is $\left.\pi\left[\theta_{\mathrm{FHWM}} / 2\right]^{2}\right)$. Thus, we reduce the mass to a fraction $\ln (2)$, and use half of the published FWHM size as the effective radius. Mueller et al. (2002) list masses for a sphere, not an aperture, and so the mass (taken for the smaller of their radii) has to be scaled up by a factor of order $\pi / 2 \approx 1.57$ (Kauffmann et al. 2008, Equation (13)). If more than one distance is listed for a given object, we adopt the smaller one (yielding a lower limit to $m[r] / m_{\text {lim }}[r]$ derived below).

\section{ANALYSIS}

\subsection{A Threshold for Massive Star Formation?}

At given radius, a cloud fragment (i.e., clump, core, etc.) can be compared against Equation (1) by deriving the mass ratio $m(r) / m_{\lim }(r)$ (where $m_{\lim }[r]=870 M_{\odot}[r / \mathrm{pc}]^{1.33}$ ), to which we refer as the "compactness." "Secondary cores" (only listed by Ragan et al. and Rathborne et al.) are suppressed by characterizing star-forming regions (i.e., a given massive star or an entire IRDC) by their most compact fragment, $\max \left[m(r) / m_{\lim }(r)\right]$.

Figure 3 gives $\max \left[m(r) / m_{\lim }(r)\right]$ as derived for the samples examined here. This is based on the mass-size data presented in Figure 2. The compactness assumes a range of values in every sample. This spread is captured by plotting several percentiles.

As suggested by Figure 2, we can clearly see in Figure 3 that regions forming massive stars are, at given radius, more massive than the limiting mass, $m_{\lim }(r)$. In all surveys of MSF regions, $>75 \%$ of the clouds have a maximum compactness $>1.7$. One

\footnotetext{
5 We further correct their masses by factors $4 /(\pi[(r / p c)$. $\left.\left.206,265^{\prime \prime} /(d / \mathrm{pc})\right]^{2} / 1^{\prime \prime} .2^{2}\right)$, where $d$ is distance, since pixels per beam (as erroneously adopted) have to be replaced by pixels per clump in Equation (5) of Ragan et al. (2009).
}

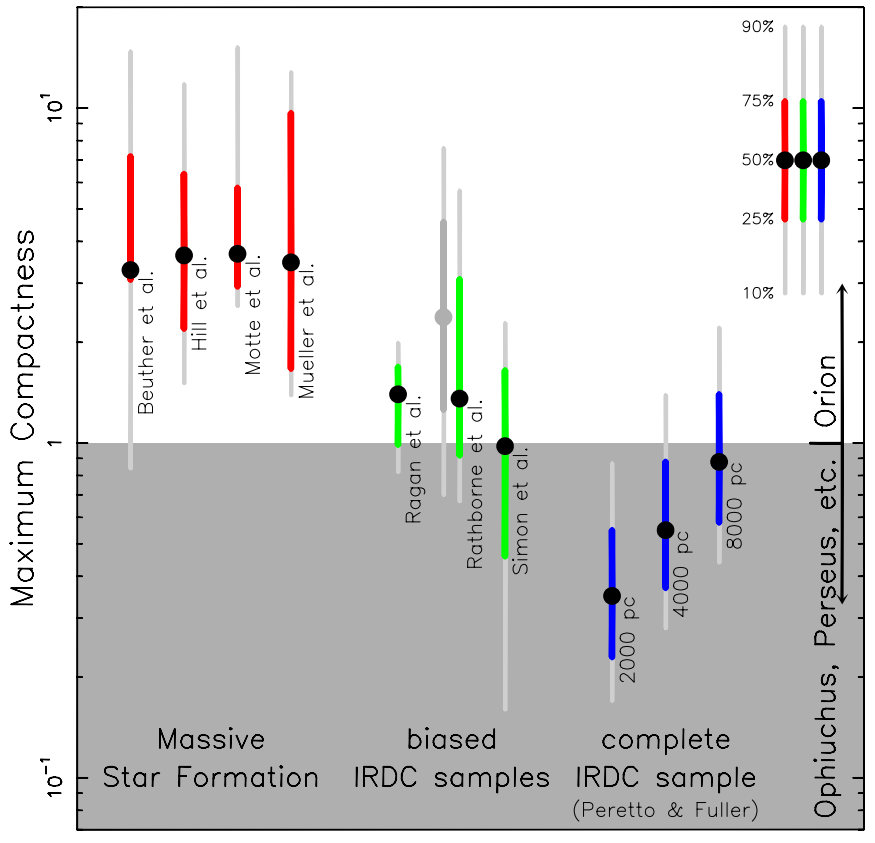

Figure 3. Percentiles of the maximum compactness per cloud, $\max [m(r) /$ $\left.m_{\mathrm{lim}}(r)\right]$, for various cloud samples. For a given sample, the ratios below which a certain fraction (e.g., 25\%) of the sample members reside are indicated by bars. Local non-MSF clouds (Figure 2(a)) have a compactness $\leqslant 1$ (Equation (1)). The bars for the Rathborne et al. (2006) sample include (left), respectively, exclude (right), their "em" cores. Clearly, the IRDCs do not reside in the mass-size space unambiguously associated with MSF.

(A color version of this figure is available in the online journal.)

survey (Beuther et al. 2002) contains a very small number of regions $(\sim 10 \%)$ less compact than required by Equation (1). These regions might be interesting targets for follow-up studies. In general, though, this analysis corroborates the hypothesis that Equation (1) approximates a threshold for MSF.

\subsection{Are IRDCs Unusually Dense and Massive?}

Figure 3 provides a compactness analysis for IRDCs. We separately characterize the Rathborne et al. (2006) sample including and excluding their "em" cores with associated $8 \mu \mathrm{m}$ sources (which are not dark). "True" IRDCs will have properties in between these extremes. Two interesting trends manifest in these $m(r) / m_{\text {lim }}(r)$ data.

First, IRDCs have masses which are, for given size, comparable to those of solar neighborhood clouds not forming massive stars (e.g., Ophiuchus and Perseus). In all samples, $\gtrsim 25 \%$ of all clouds have a compactness $<1$. Except for the Rathborne et al. (2006) clouds, $\geqslant 75 \%$ of all targets exceed Equation (1) by a factor $<2$.

Second, IRDCs are less compact than regions forming massive stars. For example, excluding the Rathborne et al. (2006) targets, $>75 \%$ of all IRDCs are less compact than most $(>75 \%)$ of the MSF regions.

In summary, the IRDCs studied here have (for given size) masses in between those of regions with and without MSF (where "true" Rathborne et al. IRDCs have properties in between the two extremes shown). Very clearly, they do not reside in the mass-size space unambiguously associated with the formation of massive stars.

However, before drawing final conclusions, let us consider some biases affecting our analysis. First, Ragan et al. (2009) 


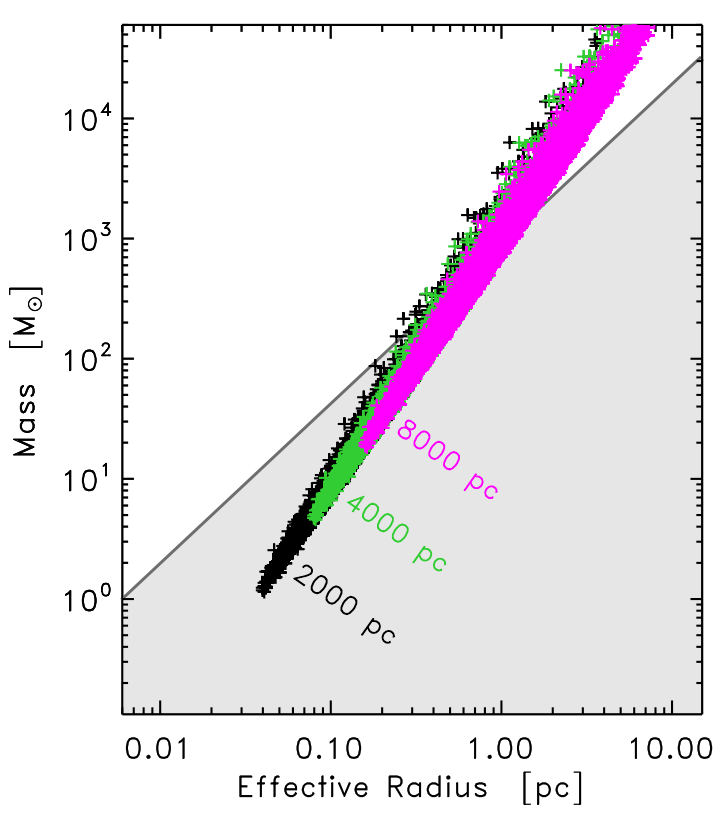

Figure 4. Same as Figure 2(c), but for the Peretto \& Fuller (2009) sample (projected out to various distances).

(A color version of this figure is available in the online journal.)

derive masses using CLUMPFIND, while Rathborne et al. (2006) use GAUSSCLUMPS. For the former, Paper I explicitly showed that the derived masses are, for given radius, just $\lesssim 70 \%$ of those derived using our dendrogram approach. For the latter, the same is expected, since the Gaussian fits only describe a fraction of the emission. In a given map, our characterization scheme from Papers I and II would thus find larger masses.

These biases are countered by other factors, though. We use the "case A" masses (assuming bright IR foregrounds) provided by Ragan et al. (2009). Following Peretto \& Fuller (2009), their "case B" (fainter foregrounds) appears to be more realistic. The masses could thus be lower by a factor $\sim 2$ (Ragan et al. 2009). Similarly, Pineda et al. (2008) suggests ${ }^{13}$ CO-to-mass conversion factors lower than used by Simon et al. (2006). In any case, similar biases affect the data for MSF regions. Differences between these and IRDCs are not likely to only come from observational uncertainties.

Finally, none of the IRDCs in the Ragan et al., Rathborne et al., and Simon et al. samples are "typical" for the general Galactic population. Ragan et al. (2009) and Rathborne et al. (2006) select clouds which are unusually dark in $8 \mu \mathrm{m}$ images. Simon et al. (2006) only characterize IRDCs which are relatively large and dark and are clearly detected in ${ }^{13} \mathrm{CO}$ emission. All this excludes IRDCs of low mass and density from the samples. Less-biased IRDC samples should thus be less compact than derived here.

\subsection{Typical Star Formation Properties in the Galaxy}

The Peretto \& Fuller (2009) catalog lists IRDC angular sizes and column densities for the entire Galactic Plane covered by Spitzer. It thus provides an ideal tool to derive a first idea of typical IRDC properties. Since they likely constitute (to our present knowledge) the typical reservoir of Galactic starforming gas, IRDC characteristics probably gauge the early state of Galactic star-forming regions.

Since no distances are known for the Peretto \& Fuller IRDCs, we constrain their masses and sizes assuming a reasonable range
Table 1

Compact Peretto \& Fuller (2009) IRDCs

\begin{tabular}{lcccc}
\hline \hline $\begin{array}{l}\text { Distance }^{\mathrm{a}} \\
(\mathrm{kpc})\end{array}$ & $\begin{array}{c}\text { Number }^{\mathrm{b}} \\
-\end{array}$ & $\begin{array}{c}\text { Fraction }^{\mathrm{c}} \\
(\%)\end{array}$ & $\begin{array}{c}\text { Mass }^{\mathrm{b}} \\
\left(10^{6} M_{\odot}\right)\end{array}$ & $\begin{array}{c}\text { Mass Fraction }^{\mathrm{c}} \\
(\%)\end{array}$ \\
\hline 2 & 831 & 7 & 2.0 & 71 \\
4 & 2218 & 20 & 9.8 & 87 \\
6 & 3639 & 32 & 23.6 & 93 \\
8 & 4778 & 42 & 43.2 & 96 \\
\hline
\end{tabular}

Notes.

${ }^{\text {a }}$ Distance to which the sample is projected.

${ }^{\mathrm{b}}$ Number of clouds with $m(r)>m_{\lim }(r)$, and their total mass.

${ }^{\mathrm{c}}$ Mass and number fraction of compact clouds.

of distances. Analysis by Simon et al. (2006) and Jackson et al. (2008) suggests that most IRDCs have distances of $2-8 \mathrm{kpc}$. Figure 4 illustrates the derived masses and sizes, and Table 1 characterizes the IRDCs found to be compact (i.e., $\left.m[r] / m_{\lim }[r]>1\right)$.

This analysis has two interesting results. First, by number, most of the Peretto \& Fuller (2009) IRDCs have masses and sizes comparable to those of solar neighborhood clouds devoid of massive stars (i.e., they are not compact). This holds even when adopting the largest reasonable distance. Second, the compact clouds contain most of the mass (more accurately: most of the area-integrated column density) seen in these IRDCs, even for small IRDC distances.

Unfortunately, the Peretto \& Fuller (2009) survey is (like most extinction studies) uncertain in the sense that it assumes that the diffuse Galactic emission can be reliably modeled in its spatial distribution. This may not be true. In this spirit, the results from this section should be taken as an indication, not as a final result.

\section{DISCUSSION}

\subsection{Mass-Size Structure of MSF Clouds}

Consider the following toy model to understand the expected mass-size properties of MSF clouds. Stars probably form on a timescale $\tau_{\mathrm{sf}}$ slower $\left(\varepsilon_{\mathrm{ff}}<1\right)$ than the free-fall timescale, ${ }^{6} \tau_{\mathrm{sf}} \gtrsim$ $\tau_{\mathrm{ff}} / \varepsilon_{\mathrm{ff}} \propto 1 /\left(\varepsilon_{\mathrm{ff}}\langle\varrho\rangle^{1 / 2}\right)$. In spherical symmetry, mass, size, and density are related by $\langle\varrho\rangle=\varepsilon_{\varrho} m /\left(4 / 3 \pi r^{3}\right)$, where $\varepsilon_{\varrho}<1$ takes line-of-sight material not associated with the sphere into account. A specific star formation timescale then requires that

$$
m(r) \gtrsim 27.1 M_{\odot} \frac{1}{\varepsilon_{\varrho} \varepsilon_{\mathrm{ff}}^{2}}\left(\frac{\tau_{\mathrm{sf}}}{10^{5} \mathrm{yr}}\right)^{-2}\left(\frac{r}{0.1 \mathrm{pc}}\right)^{3} .
$$

Further, to form a star of certain mass, $M_{\star}$, a mass reservoir larger than $M_{\star}$ is necessary:

$$
m(r) \gtrsim M_{\star} / \varepsilon_{m} .
$$

Figure 1 evaluates these limits for a star of $8 M_{\odot}$, based on efficiencies $^{7}$ and timescales from Spitzer observations of solar

\footnotetext{
$6 \tau_{\mathrm{ff}}=(3 \pi /[32 G\langle\varrho\rangle])^{1 / 2}$, where $G$ is the constant of gravity and $\langle\varrho\rangle$ is the volume-averaged density.

7 The main accretion phase of a low-mass star (IR-classes 0 and I) typically finishes after $7 \times 10^{5} \mathrm{yr}$ (Evans et al. 2009). Typical free-fall timescales of their natal cores $\sim 10^{5} \mathrm{yr}$ (Enoch et al. 2008) then imply $\varepsilon_{\mathrm{ff}} \approx 1 / 7$. Further, $\varepsilon_{\varrho} \approx 1 / 2$ (Kauffmann et al. 2008, Equation (13)) and $\varepsilon_{m} \approx 1 / 3$ (Alves et al. 2007). Since massive stars might form faster, and the star formation efficiency is not constrained well, we explore $3 \leqslant \tau_{\mathrm{sf}} / 10^{5} \mathrm{yr} \leqslant 7$ and $1 / 3 \leqslant \varepsilon_{m} \leqslant 1 / 2$ in Figure 1.
} 
neighborhood clouds. Within the model, cloud collapse will only yield a massive star if initiated inside the boundaries set by Equations (2) and (3). Krumholz \& McKee (2008) provide a similar limit, derived assuming that the collapsing region is heated by a cluster of low-mass stars (in our terminology, they use $\varepsilon_{m}=1 / 2$ ).

In order to sustain MSF, at least a few cloud fragments in MSF clouds must reside within the theoretical MSF boundaries mentioned above (Figure 1). The global structure of these clouds can usually be described by power laws, $m(r)=m_{0} \cdot r^{b}$, with $b<2$ (Paper II). Such power laws imply that MSF clouds violate $m(r)<m_{\text {lim }}(r)$ (Equation (1)). Depending on slope $(b)$, intercept $\left(m_{0}\right)$, and their interplay, such excesses are expected for radii $\gg 0.1 \mathrm{pc}$ (Figure 1). This is just what we find for MSF clouds (Figure 2(b)).

MSF is thus only possible if clouds' slopes are shallow, intercepts are large, or both, when compared to Equation (1). This permits a new way to quantitatively compare the structure of clouds with and without MSF. Pure differences in $m_{0}$ imply that MSF and non-MSF clouds only differ in their absolute properties. Differences in slopes $b$, however, imply relative differences in the structure, such as deviations in the hierarchical cloud structure.

\subsection{Average State of IRDCs}

The IRDC properties mentioned in the introduction $\left(n\left[\mathrm{H}_{2}\right] \gtrsim\right.$ $\left.10^{5} \mathrm{~cm}^{-3}, N\left[\mathrm{H}_{2}\right] \gtrsim 10^{23} \mathrm{~cm}^{-2}, m \gtrsim 10^{3} M_{\odot}\right)$ only seem to characterize the densest patches in very large and massive IRDCs. They are not well suited to describe IRDCs on average.

Some IRDCs with $m(r)<m_{\text {lim }}(r)$ might further evolve and eventually undergo MSF. And particular dust properties could, in principle, erroneously indicate $m(r)<m_{\text {lim }}(r)$ where the reverse is true. However, such caveats are not usually considered when using IRDC data to constrain MSF. Thus, we abstain from such considerations.

Our study suggests that many IRDCs, if not most, are not related to MSF. One thus has to be prudent when using IRDC properties to constrain MSF initial conditions. Most studies discussing IRDCs as pre-MSF sites concentrated on very opaque IRDCs of large angular size. These clouds often violate Equation (1), and many of them are good MSF candidates.

\subsection{Do Most Stars form in Just Few IRDCs?}

Rathborne et al. (2006) suggest that most of the Galactic star formation might come from IRDCs. The absence of other likely reservoirs of star-forming gas evinces this too. By number, most IRDCs are likely to form stars and clusters of low and intermediate mass, just as Ophiuchus and Perseus do.

Still, many IRDCs will turn toward MSF. Interestingly, Table 1 suggests that most of the mass located in IRDCs is in clouds that will form massive stars. For example, the 250 most compact clouds from the Peretto \& Fuller (2009) sample (identified assuming a common distance) contain more than $50 \%$ of the area-integrated column density of all IRDCs. This suggests that they also contain a major fraction of the mass seen in IRDCs. If this reasoning is correct, just few $10^{2}$ IRDCs (and not all $\sim 10^{4}$ : Rathborne et al. 2006) might contain most of the Galaxy's star-forming gas. Given the uncertain nature of the properties derived from the Peretto \& Fuller (2009) data (Section 3.3), this conclusion is far from certain, though.

\section{CONCLUSIONS}

This Letter studies whether Infrared Dark Clouds are able to form massive stars. Our main conclusions are as follows.

1. Observations of regions with and without massive star formation suggest that the condition $m(r) \leqslant$ $870 M_{\odot}(r / \mathrm{pc})^{1.33}$ (Equation (1)) approximates a threshold for massive star formation (Section 3.1). massive star formation clouds differ from those obeying Equation (1) in mass-size slope or intercept (Figure 1, Section 4.1).

2. Many Infrared Dark Clouds (Section 3.2), if not most (Section 3.3), do not exceed Equation (1). Without further significant evolution, such clouds are unlikely candidates for massive star formation, but they might well form stars and clusters of up to intermediate mass (like Perseus and Ophiuchus). Very opaque Infrared Dark Clouds of large angular size constitute good massive star formation candidates.

3. Provided extinction-based masses can be trusted, just few $10^{2}$ Infrared Dark Clouds might contain a major fraction of the Galaxy's star-forming gas (Section 4.3). These Infrared Dark Clouds would be dense and massive enough to host massive star formation.

We are indebted to a careful referee, who helped to significantly improve the text. This research was supported by an appointment of J.K. to the NASA Postdoctoral Program at the Jet Propulsion Laboratory, administered by Oak Ridge Associated Universities through a contract with NASA. It was excuted at the Jet Propulsion Laboratory, California Institute of Technology, under contract with the National Aeronautics and Space Administration.

\section{REFERENCES}

Abergel, A., et al. 1996, A\&A, 315, L392

Alves, J., Lombardi, M., \& Lada, C. 2007, A\&A, 462, L17

Beuther, H., Churchwell, E. B., McKee, C. F., \& Tan, J. C. 2007, in Protostars and Planets V, ed. K. Keil, B. Reipurth, \& D. Jewitt (Tucson, AZ: Univ. Arizona Press), 165

Beuther, H., Schilke, P., Menten, K. M., Motte, F., Sridharan, T. K., \& Wyrowski, F. 2002, ApJ, 566, 945

Beuther, H., \& Steinacker, J. 2007, ApJ, 656, L85

Bohlin, R., Savage, B., \& Drake, J. 1978, ApJ, 224, 132

Carey, S. J., Clark, F. O., Egan, M. P., Price, S. D., Shipman, R. F., \& Kuchar, T. A. 1998, ApJ, 508, 721

Carey, S. J., Feldman, P. A., Redman, R. O., Egan, M. P., MacLeod, J. M., \& Price, S. D. 2000, ApJ, 543, L157

Egan, M. P., Shipman, R. F., Price, S. D., Carey, S. J., Clark, F. O., \& Cohen, M. 1998, ApJ, 494, L199

Enoch, M. L., Evans, N. J., II, Sargent, A. I., Glenn, J., Rosolowsky, E., \& Myers, P. 2008, ApJ, 684, 1240

Evans, N. J., et al. 2009, ApJS, 181, 321

Hill, T., Burton, M. G., Minier, V., Thompson, M. A., Walsh, A. J., HuntCunningham, M., \& Garay, G. 2005, MNRAS, 363, 405

Jackson, J. M., Finn, S. C., Rathborne, J. M., Chambers, E. T., \& Simon, R. 2008, ApJ, 680, 349

Kauffmann, J., Bertoldi, F., Bourke, T., Evans, N., II, \& Lee, C. 2008, A\&A, 487, 993

Kauffmann, J., Pillai, T., Shetty, R., Myers, P. C., \& Goodman, A. A. 2010a, ApJ, 712, 1137

Kauffmann, J., Pillai, T., Shetty, R., Myers, P. C., \& Goodman, A. A. 2010b, ApJ, 716,433

Krumholz, M. R., \& McKee, C. F. 2008, Nature, 451, 1082

Menten, K. M., Pillai, T., \& Wyrowski, F. 2005, in IAU Symp. 227, Massive Star Birth: A Crossroads of Astrophysics, ed. E. Churchwell et al. (Cambridge: Cambridge Univ. Press), 23 
Motte, F., Bontemps, S., Schilke, P., Schneider, N., Menten, K. M., \& Broguière, D. 2007, A\&A, 476, 1243

Mueller, K. E., Shirley, Y. L., Evans, N. J., II, \& Jacobson, H. R. 2002, ApJS, 143,469

Ossenkopf, V., \& Henning, T. 1994, A\&A, 291, 943

Perault, M., et al. 1996, A\&A, 315, L165

Peretto, N., \& Fuller, G. A. 2009, A\&A, 505, 405

Pillai, T., Wyrowski, F., Menten, K., \& Krügel, E. 2006, A\&A, 447, 929

Pineda, J., Caselli, P., \& Goodman, A. 2008, ApJ, 679, 481

Ragan, S., Bergin, E., \& Gutermuth, R. 2009, ApJ, 698, 324
Rathborne, J. M., Jackson, J. M., Chambers, E. T., Simon, R., Shipman, R., \& Frieswijk, W. 2005, ApJ, 630, L181

Rathborne, J. M., Jackson, J. M., \& Simon, R. 2006, ApJ, 641, 389

Rathborne, J. M., Simon, R., \& Jackson, J. M. 2007, ApJ, 662, 1082

Rosolowsky, E., Pineda, J., Kauffmann, J., \& Goodman, A. 2008, ApJ, 679, 1338

Rygl, K. L. J., Wyrowski, F., Schuller, F., \& Menten, K. M. 2010, A\&A, 515, A42

Simon, R., Rathborne, J. M., Shah, R. Y., Jackson, J. M., \& Chambers, E. T. 2006, ApJ, 653, 1325 